\title{
Philosophiques
}

\section{Comme une suite intemporelle de chocs. Adorno et l'expérience moderne en crise}

\section{Pascale Cornut St-Pierre}

Volume 37, numéro 2, automne 2010

URI : https://id.erudit.org/iderudit/045192ar

DOI : https://doi.org/10.7202/045192ar

Aller au sommaire du numéro

Éditeur(s)

Société de philosophie du Québec

ISSN

0316-2923 (imprimé)

1492-1391 (numérique)

Découvrir la revue

Citer cet article

Cornut St-Pierre, P. (2010). Comme une suite intemporelle de chocs. Adorno et l'expérience moderne en crise. Philosophiques, 37(2), 457-473.

https://doi.org/10.7202/045192ar
Résumé de l'article

" La vie s'est transformée en une suite intemporelle de chocs ", écrivait Adorno, dans ses Minima Moralia, pour décrire l'expérience du front lors de la Seconde Guerre mondiale. À partir de la notion de choc, qui ponctue l'ensemble de l'oeuvre d'Adorno, se dégage en fait une problématique plus générale d'une crise de l'expérience moderne. En mettant en évidence certains parallèles entre les thèmes de la philosophie d'Adorno et la théorie psychanalytique du choc traumatique, j'entends cerner avec plus de précision la teneur de la crise dont il est question, ses conséquences, ainsi que les possibilités qu'entrevoit le philosophe pour y mettre un terme. 


\title{
Comme une suite intemporelle de chocs. Adorno et l'expérience moderne en crise
}

\author{
PASCALE CORNUT ST-PIERRE \\ Université de Montréal \\ pascale.cornut.st-pierre@umontreal.ca
}

\begin{abstract}
RÉSUMÉ. - «La vie s'est transformée en une suite intemporelle de chocs», écrivait Adorno, dans ses Minima Moralia, pour décrire l'expérience du front lors de la Seconde Guerre mondiale. À partir de la notion de choc, qui ponctue l'ensemble de l'œuvre d'Adorno, se dégage en fait une problématique plus générale d'une crise de l'expérience moderne. En mettant en évidence certains parallèles entre les thèmes de la philosophie d'Adorno et la théorie psychanalytique du choc traumatique, j'entends cerner avec plus de précision la teneur de la crise dont il est question, ses conséquences, ainsi que les possibilités qu'entrevoit le philosophe pour y mettre un terme.
\end{abstract}

ABSTRACT. - Reflecting upon the experience of the battle line during the Second World War, Adorno claimed that "life has changed into a timeless succession of shocks". From this concept of shock, which punctuates the whole of Adorno's work, emerges the more general problem of the crisis of modern experience. By drawing parallels between aspects of Adorno's philosophy and of the psychoanalytic theory of traumatic shock, I intend to define more precisely the content of this crisis, its consequences, and the possibilities for its resolution glimpsed by the philosopher.

Le Temps et l'Espace sont morts hier. Nous vivons déjà dans l'absolu, puisque nous avons déjà créé l'éternelle vitesse omniprésente. MarinetTI, Premier manifeste du futurisme'.

L'homme moderne, à en croire Adorno, paierait les multiples progrès qui agrémentent son existence d'un prix dont il ne se rendrait que très mal compte. Outre une puissance technique et sociale sans précédent, l'avancée des lumières de sa raison lui vaudrait aussi une inaptitude croissante à faire l'expérience du monde qui l'entoure. Ce qui jadis se présentait à lui dans une continuité harmonieuse l'assaillirait dorénavant comme une incompréhensible succession de chocs. Autour de cette notion de choc, qui ponctue l'ensemble de l'œuvre d'Adorno, s'esquisse toute une problématique d'une crise de l'expérience, qui serait, de plus, typiquement moderne et dont la résolution serait cruciale pour l'avenir de l'humanité. L'affirmation a de quoi faire sourciller et appelle quelque explication: nous, modernes, serions donc

1. Marinetti, Filippo Tommaso, "Premier manifeste du futurisme ", Tuons le clair de lune! Manifestes futuristes et autres proclamations, Paris, Mille et une nuits, 2005, p. 12. 
devenus inaptes à l'expérience? Le propos d'Adorno, me semble-t-il, ne relève pas d'un romantisme nostalgique du temps d'avant, et n'est pas non plus que métaphorique. Le propos prend plutôt les allures d'un constat clinique. Freud, à cet égard, fait figure de modèle exemplaire pour l'interprétation d'Adorno: n'avait-il pas déjà souligné, dès les années suivant la Grande Guerre, l'immense difficulté des individus ayant subi un choc traumatique à prendre pleinement conscience de leur expérience traumatisante? Les chocs dont traite Adorno s'apparentent, pour ainsi dire, à des chocs traumatiques de masse, c'est-à-dire à des chocs quotidiens et systématisés. La crise de l'expérience qu'ils induisent aurait alors à voir, comme dans l'hypothèse psychanalytique, avec un certain trouble de la mémoire et de l'oubli, ainsi qu'avec un nouveau rapport - ou avec la destruction de tout rapport - au temps et à l'espace. Le temps et l'espace sont morts hier, écrivait fougueusement Marinetti, il y a près d'un siècle - peut-être avait-il raison, mais peutêtre n'était-ce pas aussi émancipateur qu'il le croyait...

La guerre faisant figure d'événement traumatisant par excellence, il n'est pas étonnant qu'elle soit à la source de maintes réflexions sur ce que je n'ai encore cerné que vaguement sous le vocable de crise de l'expérience. Outre Freud, que l'analyse des névroses de guerre mena à remanier substantiellement ses vues théoriques sur le psychisme, Walter Benjamin fait partie de ces penseurs dont la guerre aiguillonna les réflexions sur l'expérience, et dont Adorno s'est très manifestement inspiré. Dans son essai intitulé Le narrateur, Benjamin constate le déclin généralisé de l'art de raconter et, le mettant au compte d'une perte de la faculté même de faire l'expérience des choses et des événements, en situe l'origine lors la Première Guerre mondiale: "N'avions-nous pas constaté, après l'Armistice, que les combattants revenaient muets du front, non pas plus riches, mais plus pauvres d'expérience communicable ${ }^{2}$ ?" C'est un constat que reprendra Adorno presque mot pour mot dans Minima Moralia, dans le long aphorisme intitulé "Hors de portée ». Adorno en radicalise cependant le propos: si la démesure des forces en présence lors de cette Première Guerre a rendu une véritable expérience de ce qui s'y passa très difficile, le progrès des vingt années qui la séparèrent de la Seconde a rendu cette dernière "absolument au-delà de toute expérience ${ }^{3} »$. En se référant toujours à la Seconde Guerre mondiale, Adorno poursuit:

Partout, avec chaque explosion, elle a forcé l'écran protecteur des réactions sensorielles, derrière lequel peut se constituer l'expérience, c'est-à-dire la durée

2. Walter Benjamin, «Le narrateur. Réflexions sur l'œuvre de Nicolas Leskov », CEuvres II - Poésie et Révolution, Paris, Denoël, 1971, p. 140.

3. Theodor W. Adorno, Minima Moralia. Réflexions sur la vie mutilée, Paris, Payot et Rivages, 2003, p. 70. 
qui s'écoule entre l'oubli salutaire et le souvenir salutaire. La vie s'est transformée en une suite intemporelle de chocs entre lesquels il y a des trous béants, des intervalles vides et paralysés. Or il n'y a rien peut-être de plus funeste pour l'avenir que le fait qu'à proprement parler bientôt plus personne ne sera en mesure de penser encore à cette guerre, car tout traumatisme et tout choc non surmonté chez ceux qui en reviennent est un germe de destruction à venir ${ }^{4}$.

Cette guerre sera non seulement inénarrable, comme le disait déjà Benjamin à propos de la Première, mais échappera même, selon Adorno, aux efforts de ceux qui y participaient à se la remémorer comme expérience vécue. Ce passage est le véritable point de départ de la réflexion du présent essai: il livre en condensé tous les éléments qui y seront développés. La vie n'est plus la vie telle qu'on pouvait la concevoir autrefois, mais désormais une "suite intemporelle de chocs"; la cause réside dans cet "écran protecteur », qui fut rompu par la démesure des forces à l'œuvre; l'expérience, ce qui, dit-on, nous est devenu inaccessible, y est définie comme "la durée qui s'écoule entre l'oubli salutaire et le souvenir salutaire»; et finalement, cette idée inquiétante selon laquelle le traumatisme est " un germe de destruction à venir »...

Ces tournures utilisées par Adorno résonnent d'un écho qui sera familier au lecteur ayant quelque connaissance de l'œuvre de Freud. Dans $A u$ delà du principe de plaisir, ce dernier s'interroge sur la genèse des névroses de guerre et, plus généralement, de ce que les milieux médicaux de l'époque nomment communément les névroses traumatiques - que l'on désigne, dans le jargon contemporain, par "syndrome de stress post-traumatique". Freud relève d'abord ce qui lui paraît constituer le facteur déterminant du traumatisme, soit l'absence de préparation à l'événement traumatisant, le facteur surprise, l'effroi. Il note ensuite l'apparente indifférence des patients, à l'état de veille, au souvenir de leur accident, mais la récurrence des cauchemars qui, la nuit venue, leur font revivre l'événement traumatisant et ressentir de nouveau cet effroi terrible. Afin d'expliquer ce phénomène, Freud émet l'hypothèse d'une sorte de compulsion de répétition, qui serait psychiquement plus originaire que le principe de plaisir.

Pour étayer son hypothèse, Freud trace un portrait minutieux, inspiré d'une métaphore neurologique, de ce qu'il appelle pour l'occasion la «substance psychique». Il imagine une vésicule originaire de substance excitable, au sein de laquelle l'énergie laisse des traces durables - ce sont les origines de la mémoire - qui forment comme autant de canaux par lesquels l'énergie pourra ensuite repasser, endiguée - ce repassage seul pouvant devenir

4. Ibid. 
conscient $^{5}$. Mais l'énergie finirait par brûler, littéralement, cet être vivant rudimentaire, si ce dernier ne se dotait pas d'une sorte de pare-excitations. À ce sujet, Freud écrit :

Ce petit fragment de substance vivante est plongé dans un monde extérieur chargé des énergies les plus fortes et il succomberait sous le coup des excitations qui en proviennent s'il n'était pourvu d'un pare-excitations qu'il acquiert ainsi: sa couche la plus superficielle abandonne la structure propre au vivant, devient dans une certaine mesure anorganique et fonctionne désormais comme une enveloppe ou membrane spéciale qui tient l'excitation à l'écart: les énergies du monde extérieur ne peuvent ainsi transmettre qu'un fragment de leur intensité aux couches voisines qui sont restées vivantes ${ }^{6}$.

Cette couche superficielle, brûlée par les énergies du monde extérieur, incapable d'être modifiée davantage et donc d'enregistrer quoi que ce soit, serait en outre le lieu de la conscience - là où les excitations passent sans laisser de trace durable. À la suite de ce développement, Freud qualifiera de traumatiques les excitations externes qui sont assez fortes pour faire effraction à cette barrière psychique - ou, pour reprendre les termes d'Adorno, pour forcer cet écran protecteur. Le traumatisme signifierait ainsi l'intrusion, au sein du système psychique, d'une quantité d'énergie plus grande que celle qui pouvait être prise en charge, et ses symptômes manifesteraient les vains efforts du psychisme pour neutraliser, après coup, ce dommageable surplus d'énergie. Le caractère répétitif des rêves des patients traumatisés refléterait le besoin de renforcer rétroactivement, par l'angoisse, cette barrière de protection qui céda par manque de préparation face à ce qui allait l'assaillir.

Si l'on reprend maintenant le passage cité précédemment, où Adorno parle de cette guerre qui, de toute part, a forcé l'écran protecteur de ceux qui y étaient, et qui en outre viendra hanter durablement l'humanité, l'allusion à Freud devient évidente. Il est possible, de plus, d'éclairer la définition énigmatique que donne Adorno de cette expérience qu'aurait rendu possible l'écran protecteur du psychisme, soit l'expérience comme «durée qui s'écoule entre l'oubli salutaire et le souvenir salutaire ${ }^{7} »$. La psychanalyse, dès ses balbutiements, a conçu la névrose comme trouble du rapport entre la mémoire et la conscience, comme incapacité, de la part du malade, tant d'oublier que de se remémorer l'origine de son mal — «c'est

5. Sigmund Freud, "Au-delà du principe de plaisir ", Essais de psychanalyse, Paris, Payot \& Rivages, 2001, p. 72 et suivantes. Freud reprend là une idée qu'il avait déjà formulée quelque vingt ans plus tôt dans son Esquisse d'une psychologie scientifique, œuvre de jeunesse abandonnée et publiée seulement à titre posthume. C'est un développement que reprend aussi Benjamin lorsque, dans son essai sur Baudelaire, il entreprend sa réflexion sur "l'expérience vécue du choc». Benjamin, "Sur quelques thèmes baudelairiens", Euvres II, p. 230 et suivantes.

6. Freud, "Au-delà du principe de plaisir", p. 75.

7. Adorno, Minima Moralia, p. 70. 
de réminiscences surtout que souffre l'bystérique " ", écrivaient, déjà en 1895, Freud et Breuer. Lors d'un traumatisme, l'événement marquant s'inscrit directement dans le psychisme, sans nécessairement passer par la conscience, son influence étant, dans ce dernier cas, d'autant plus profonde: l'individu se retrouve alors complètement démuni face à ce qui le ronge de l'intérieur, mais qu'il est impuissant à comprendre. C'est même l'absence tant de l'oubli salutaire que du souvenir salutaire qui provoquerait cet éternel retour du même (c'est l'expression qu'il utilise ${ }^{9}$ ) que Freud voit dans la compulsion de répétition. D'abord amenée à son attention par la récurrence des rêves traumatiques, Freud la reconnaît ensuite dans ce qu'il appelle la névrose de transfert: le patient, incapable de se souvenir de ce qui, en lui, est refoulé, se verra obligé, lors du traitement psychanalytique, "de répéter le refoulé comme expérience vécue dans le présent au lieu de se le remémorer comme un fragment du passé p $^{10}$.

Le but des développements qui précèdent n'est pas, loin de là, de réduire le propos d'Adorno à une plate répétition des théories psychanalytiques. Les propos de Freud me semblent cependant susceptibles de mettre en lumière certaines affirmations fulgurantes d'Adorno, et d'expliciter ce qui risquerait, sinon, de rester fort obscur. Un dernier passage — probablement le plus important - de la citation de Minima Moralia que j'ai présentée comme point de départ de la présente réflexion reste à développer : l'affirmation selon laquelle la vie se serait muée en «une suite intemporelle de chocs $^{11}$ ". S'il ne livrera cette fois aucune solution, Freud peut néanmoins encore donner un dernier indice. Après avoir présenté ses hypothèses sur la genèse de la conscience par le biais de cette vésicule de substance excitable dont j'ai déjà parlé, Freud réaffirme, en polémique avec la conception kantienne de l'espace et du temps, le caractère intemporel des processus psychiques primordiaux, inconscients. L'ordonnancement temporel de la réalité ne serait, selon lui, propre qu'aux faits psychiques conscients, et pour cette raison ne serait que dérivé et secondaire; il ajoute ensuite, énigmatiquement, que " $[\mathrm{d}]$ ans ce mode de fonctionnement du système [conscient] on pourrait trouver un autre mode de pare-excitations ${ }^{12}{ }^{\prime}$. L'organisation spatio-temporelle de nos affections jouerait-elle donc, comme le laisse entendre Freud, un rôle protecteur à l'égard de l'expérience?

C'est à cette question, notamment, que répond Walter Benjamin dans son essai intitulé Sur quelques thèmes baudelairiens, au cours de sa réflexion

8. Sigmund Freud et Joseph Breuer, Études sur l'hystérie, Paris, PUF, 1956, p. 5.

9. Freud, «Au-delà du principe de plaisir », p. 68.

10. Ibid., p. 64.

11. Adorno, Minima Moralia, p. 70. (Je souligne.)

12. Freud, «Au-delà du principe de plaisir », p. 77. (Je souligne.) 
sur ce qu'il nomme l' «expérience vécue du choc». Constatant l'opposition de nature qu'énonce Freud entre mémoire - empreinte psychique durable - et conscience - passage éphémère et infiniment renouvelable des sensations - , il tente de prendre la mesure de cette idée selon laquelle la conscience serait une sorte de barrage protecteur contre les influx provenant du monde extérieur. Dans un monde où se sont multipliés les stimuli sensoriels - la grande ville étant à cet égard érigée par Benjamin en figure emblématique de notre monde moderne -, l'individu doit subir un certain entraînement pour résister à ces chocs, pour atténuer leurs influences traumatisantes. Benjamin écrit à ce propos :

À mesure que l'élément de choc se fait davantage sentir dans les impressions singulières, il faut que la conscience se défende de façon plus continue contre l'excitation; mieux elle y réussit et moins les impressions particulières pénètrent dans l'expérience [Erfahrung], mais plus important aussi devient, par là même, le rôle de l'expérience vécue [Erlebnis]. Finalement on pourrait dire que la défense contre le choc a pour résultat spécifique d'assigner à l'événement - au détriment de l'intégrité même de ses contenus — une situation temporelle précise dans la conscience ${ }^{13}$.

L'ordonnancement temporel des stimuli dans le champ de la conscience aurait donc bien, si l'on en croit Benjamin, un rôle protecteur, mais cette protection se ferait au détriment même de l'expérience véritable. En effet, la temporalité consciente, lorsqu'elle s'érige en pare-excitations, éviterait non seulement le traumatisme que pourrait causer le choc, mais éviterait en même temps à l'individu d'être marqué par l'objet de son expérience. Ainsi, en même temps qu'ils auraient renforcé leurs défenses conscientes, les individus modernes auraient aussi «liquidé leur mémoire $^{14}{ }^{1} \ldots$

C'est à une semblable liquidation que renvoie, selon les termes utilisés par Benjamin, la dégradation de l'expérience véritable (Erfahrung) en une expérience simplement vécue (Erlebnis). L'expérience véritable consisterait en un contact avec ce qu'ont de concret et de singulier les choses et les événements, avec leur aura, contact qui ne laisse pas indemne le sujet de l'expérience. L'aura était définie, dans L'œuvre d'art à l'époque de sa reproductibilité technique, comme le hic et nunc d'un objet, son «ici-maintenant ", socle de son unicité et de son authenticité ${ }^{15}$; dans son essai sur Baudelaire, Benjamin ajoute que " [s]entir l'aura d'une chose, c'est lui conférer le pouvoir de lever les yeux ${ }^{16} »-$ le pouvoir de réagir à notre regard et de nous affecter en retour. Se dégage ici toute une historicité propre à l'expérience, une temporalité liée aux contenus significatifs de l'expérience et aux modifications que

13. Benjamin, «Sur quelques thèmes baudelairiens », p. 234.

14. Ibid., p. 255.

15. Benjamin, L'œuvre d'art à l'époque de sa reproductibilité technique, Paris, Éditions Allia, 2006, p. 13.

16. Benjamin, "Sur quelques thèmes baudelairiens", p. 268. 
ceux-ci induisent au sein du sujet ${ }^{17}$. C'est contre une telle historicité que se dresse l'expérience simplement vécue, l'Erlebnis: en assignant à chaque événement ou, pour reprendre la métaphore neurologique, à chaque excitation un emplacement précis dans un "temps homogène et vide ${ }^{18}$ ", le temps linéaire de la conscience, l'expérience vécue laisse de côté le contenu singulier de l'expérience, sa signification propre ${ }^{19}$. L'histoire se réduit alors à une pure succession d'unités standardisées, sans plus de connexions intimes les unes avec les autres: simple suite de chocs. Dans sa réflexion sur ce qu'il appelle l'expérience vécue $d u$ choc, Benjamin rejoint ainsi à son tour le thème de la répétition: non plus, comme chez Freud, la répétition d'un refoulé, mais la récurrence de vécus sans contenu, qui ne laissent pas de trace, et que rien ne peut plus distinguer les uns des autres - "éternel recommencement à partir de zéro ${ }^{20}$ ", écrit Benjamin.

Mine de rien, ce sont bien deux conceptions distinctes du «choc» qui se sont dégagées au cours de l'étude qui précède, deux conceptions qui formeront comme deux pans complémentaires dans la réflexion d'Adorno sur le sujet. Le choc est perçu chez Freud comme un événement générateur de traumatisme, comme quelque chose qui déborde les capacités d'expérience consciente de l'individu et qui rompt ses défenses habituelles en s'inscrivant directement dans son psychisme inconscient. Quand il traite d'un choc vécu, Benjamin décrit au contraire l'absence d'empreinte durable laissée par l'événement singulier, par suite du renforcement de cet écran protecteur qui doit protéger l'individu de ce qui lui est extérieur. Les deux analyses convergent néanmoins autour du constat suivant: face au choc, l'individu subit une perte d'expérience - que cette perte soit comprise comme incapacité de maîtriser consciemment des représentations douloureuses, ou comme inaptitude à saisir les choses dans leur singularité et à évoluer grâce à elles. Dans les deux cas, aussi, cette perte d'expérience se traduit directement par l'érosion de ce que l'on pourrait nommer le «sens historique" de l'individu, soit sa faculté de penser sa propre histoire et de se concevoir lui-même à la fois comme devenu et comme devenir. Dans les deux cas, l'histoire tend à se résorber en une stricte répétition du même.

17. C'est une telle historicité interne de l'expérience que souligne aussi Gadamer par la belle formule d'Eschyle, pathei mathos: instruit par la souffrance. Hans-Georg Gadamer, Vérité et Méthode, Paris, Seuil, 1996, p. 379.

18. Benjamin, «Thèses sur la philosophie de l'histoire» dans Euvres II, p. 285.

19. "Rather than experiences being turning points and origins which give sense to what follows them, sense is given to experience by placing each event in a narrative causal order ", écrit Jay M. Bernstein à propos de ce passage de l'Erfahrung à l'Erlebnis. Bernstein, Adorno. Disenchantment and Ethics, Cambridge, Cambridge University Press, 2001, p. 119.

20. Benjamin, "Sur quelques thèmes baudelairiens", p. 257. 
Le choc se présente aussi chez Adorno à la fois comme ce qui est incommensurable à toute expérience individuelle et comme l'abstraction qui ôte aux objets toute qualité pouvant les rendre significatifs pour le sujet. Ces deux aspects, la démesure et l'abstraction, ne sont pas immédiatement équivalents, mais deviennent étroitement liés au cours de la réflexion d'Adorno, notamment celle sur le rapport antagonique qu'entretiennent l'individu et la société. Mais avant de développer cela plus avant, j'aimerais poursuivre l'étude du fragment de Minima Moralia que j'ai déjà cité, l'aphorisme intitulé "Hors de portée ». Toujours en rapport avec la Deuxième Guerre mondiale, Adorno y identifie concrètement deux situations génératrices de chocs, soit la prépondérance de la technique et de l'information médiatique. Sur la technique, d'abord, Adorno écrit:

[L]e rythme mécanique détermine entièrement le comportement des hommes vis-à-vis de la guerre elle-même, non seulement dans la disproportion existant entre la force physique des individus et l'énergie que peuvent déployer les éléments motorisés, mais aussi jusque dans les profondeurs intimes du vécu. [...] [C]ette Seconde Guerre est absolument au-delà de toute expérience, tout autant que peut l'être la marche d'une machine par rapport aux mouvements du corps, lequel ne se met à ressembler à la machine que quand il se trouve dans un état pathologique ${ }^{21}$.

La technique semble ici problématique à cause de sa disproportion, de son hybris, certes, mais aussi et peut-être surtout parce qu'elle va jusqu'à déterminer «les profondeurs intimes » de l'individu, qui s'adapte à son rythme mécanique et se met lui-même à "ressembler à la machine». Tout se passe comme si l'individu, impuissant au milieu des forces démesurées qui se déchaînent autour de lui, est condamné à s'oublier lui-même dans sa singularité afin de s'identifier aux forces en présence, et de se considérer comme partie de la grande mécanique guerrière. Cette analyse rappelle certains passages de Marx sur le machinisme, où celui-ci constatait que le véritable sujet du processus de travail en manufacture était en réalité l'ensemble des machines assemblées en système, parmi lesquelles les travailleurs individuels ne faisaient plus figure que d'organes partiels d'un travailleur mécanique global ${ }^{22}$. Chez Marx, la disproportion de l'activité de la manufacture par rapport à celle du

21. Adorno, Minima Moralia, p. 70.

22. En plus de mettre en évidence la déshumanisation que fait subir la manufacture au travailleur, Marx souligne toute la discipline nécessaire pour y parvenir. Il cite ironiquement Andrew Ure, économiste anglais: "Dans la fabrique automatique, la difficulté essentielle réside dans la discipline nécessaire pour faire renoncer les hommes à l'irrégularité de leurs habitudes de travail et les identifier à la régularité immuable du grand automate. (Ure, cité par Marx, Le Capital, livre I, Paris, PUF, coll. Quadrige, 1993, pp. 475-476.) Benjamin à son tour reprend Marx pour mettre l'accent sur ce dressage que requiert le passage d'un travail concret à cet «ensemble de réflexes mécaniques que la machine met en jeu chez le travailleur », qui constitue un aspect important de l'expérience moderne du choc. (Benjamin, "Sur quelques thèmes baudelairiens", p. 253.) 
travailleur individuel et le caractère abstrait que revêtait pour l'ouvrier son propre travail étaient deux faces d'un même phénomène, la division technique du travail. En effet, le travail réduit à la répétition incessante d'un même geste très simple est objectivement devenu abstrait — incapable de produire par lui-même quelque valeur d'usage que ce soit — et ne gagne sa concrétude qu'à une échelle qui échappe de loin à l'individu — au terme seulement de la chaîne de montage. Le phénomène que décrit Adorno à propos du rythme mécanique de la guerre semble pouvoir se comprendre d'une façon similaire: l'individu dans la guerre ne peut plus rendre intelligibles ni les événements qui le touchent ni même ses propres actions - ces événements et ces actions ne possédant plus en eux-mêmes leur véritable sens - à moins qu'il ne fasse abstraction de sa propre expérience pour adopter le point de vue du processus général, qui par ailleurs échappe complètement à son emprise. En imitant le rythme de la machine, l'homme souhaite préserver l'intelligibilité de son monde, mais il ne le fait qu'au prix de l'oubli de lui-même.

Cette prévalence du point de vue global sur l'expérience individuelle expliquerait en partie le gigantesque essor des médias au cours du siècle dernier. La Deuxième Guerre mondiale, selon Adorno, se serait en fait jouée comme un "documentaire monstre ${ }^{23}$ ", où chacun ne comprenait finalement son rôle qu'en voyant la place qu'il occupait à l'écran. La surabondance de l'information prodiguée au public allait de pair avec l'inconscience des acteurs et traduisait «un assèchement de l'expérience, un vide qui s'est creusé entre les hommes et la fatalité qui les entraîne, en quoi réside proprement la Fatalité ${ }^{4}$ ». Rien, dans l'information journalistique, ne permet de saisir les événements dans leur singularité et dans leur relation intime avec ceux qui les vivent, ni de reconstituer la trame signifiante de leur déroulement. Benjamin écrivait déjà à ce sujet que le dessein de la presse était «de présenter les événements de telle sorte qu'ils ne puissent pénétrer dans le domaine où ils concerneraient l'expérience du lecteur ${ }^{25}$ ». Les nouvelles se succèdent alors comme autant de chocs inassimilables pour l'individu, mais néanmoins nécessaires pour qu'il puisse se doter d'une représentation générale de la situation qui l'enserre et qu'il échoue à comprendre.

La réflexion qui, tant chez Freud que chez Benjamin, se présentait avant tout comme une sorte de psychologie spéculative ${ }^{26}$, gagne désormais,

23. Adorno, Minima Moralia, p. 71.

24. Ibid.

25. Benjamin, «Sur quelques thèmes baudelairiens», p. 229. Benjamin développe aussi cette réflexion sur l'information, qu'il oppose à la narration, dans son essai sur Nicolas Leskov, «Le narrateur », pp. 145-146.

26. C'est l'expression qu'utilise Bernstein pour caractériser la démarche de Benjamin (Bernstein, Adorno. Disenchantment and Ethics, p. 118); Freud lui-même caractérise aussi sa propre entreprise de spéculation («Au-delà du principe de plaisir», p. 71). 
sous la plume d'Adorno, une épaisseur sociologique plus manifeste. Qui veut rendre compte des chocs de la vie moderne, de ses traumatismes et de son devenir abstrait, doit considérer selon Adorno les rapports qui unissent de façon conflictuelle la société et l'individu. Ce conflit marque profondément l'individu et, comme l'a bien montré la psychanalyse, c'est précisément lui qui, à la fois, lui donne forme et le divise. Dans La psychanalyse révisée, Adorno livre une explication éclairante de sa conception du choc:

Qui critique la société présente [...] ne peut pas se refuser à voir qu'elle est vécue en chocs, en coups soudains et abrupts, dus à l'aliénation de l'individu par rapport à la société $[\ldots]$. Le caractère $[\ldots]$ est dans une beaucoup plus large mesure l'effet de tels chocs que d'une expérience continuelle. Sa totalité est fictive: on pourrait presque l'appeler un système de cicatrices qui ne sont intégrées, mais jamais complètement, que dans la souffrance ${ }^{27}$.

Les chocs, et ici Adorno ne pourrait pas être plus explicite, sont le fruit d'une situation où la société reste étrangère aux individus qui la composent. Cette société marque, blesse les individus, qui ne s'individualisent en fait que par la gestion de leurs traumatismes, par la constitution d'un imparfait "système de cicatrices ". L'expérience pourrait alors être définie comme la reconnaissance des chocs subis, des chocs qui appartiennent à un sujet $m o i-$ et qui forment son histoire. Ou encore, pour reprendre une figure utilisée plus tôt, l'expérience consisterait en la douloureuse remémoration des chocs subis, remémoration salutaire qui éviterait l'incessante répétition de l'événement traumatisant.

Si cette interprétation est juste, le choc est une notion beaucoup plus fondamentale qu'il n'apparaissait au départ: il est omniprésent et constitutif de l'expérience telle qu'on la connaît - au point qu'une expérience qui en serait dépourvue puisse être décrite comme "un idéal qui ne pourrait être réalisé que dans une société non traumatique ${ }^{28} »$. La crise de l'expérience, dans ces conditions, ne saurait être qu'une simple expérience aux prises avec des chocs - c'est le cas de toute expérience -, mais se révèle plutôt être une incapacité de faire la synthèse de ces chocs, de les reconnaître, de se les remémorer. Cette incapacité, si l'on prend appui sur le modèle psychanalytique, signifierait en même temps l'incapacité de s'ériger soi-même en sujet.

Il est possible de cerner plus concrètement ce que signifie cette crise de l'expérience, ainsi que son caractère typiquement moderne, en abordant l'étude que fait Adorno de la modernité par le biais de ce qu'il appelle le culte du nouveau. Le nouveau à tout prix, la sensation forte recherchée pour elle-même, inlassablement, serait symptomatique d'un choc qui aurait désormais été érigé en bien de consommation ${ }^{29}$. «Le nouveau, écrit Adorno, une sorte de blanc dans la conscience, attendu pour ainsi dire les yeux

27. Adorno, La psychanalyse révisée, Paris, Éditions de l'Olivier, 2007, p. 21.

28. Ibid.

29. Adorno, Minima Moralia, p. 317. 
fermés, semble être la formule qui permet d'extraire de l'horreur et du désespoir leur part de stimulant ${ }^{30}$.» Dans un monde où le nouveau incarne en lui-même une valeur, le choc n'est plus quelque chose à intégrer dans la douleur, mais devient plutôt une secousse divertissante, et le contraire d'une occasion de réflexion sur soi-même et sur ce qui choque. Dans ces conditions, le choc n'est pas moins traumatique qu'avant - au contraire de ce que pouvait laisser entendre une analyse comme celle de Benjamin -, mais achève de faire éclater la structure du sujet, qui était en quelque sorte le support nécessaire de la douleur. Traumatisme et devenir abstrait du monde se rejoignent: l'individu bombardé de nouveau n'est plus apte ni à se saisir lui-même comme unité cohérente ni à percevoir l'unicité ou la qualité de ce qui l'affecte. La voie de l'éternelle répétition est libre. Adorno écrit:

Le nouveau recherché pour lui-même, produit pour ainsi dire en laboratoire, pétrifié en un schéma conceptuel, devient, dans sa brusque apparition, retour inéluctable de l'ancien, assez comparable aux névroses traumatiques. Aveuglé, l'homme voit se déchirer le voile de la succession temporelle et il découvre les archétypes de ce qui est toujours semblable [...] La décomposition du sujet s'accomplit dans son abandon au toujours identique toujours autre ${ }^{31}$.

Le nouveau, réduit à l'état de choc consommé, a perdu tout contenu qui lui aurait permis d'apporter une nouveauté effective, et ne reste qu'une pure forme, toujours la même: un désir qui s'épuise, qui ne sait plus atteindre ses objets, le désir d'un désir. L'extrême pauvreté au sein même du foisonnement de l'industrie culturelle serait l'indice de cet épuisement: on désire autre chose, mais on a oublié ce qu'autre chose pouvait être. À au moins deux reprises ${ }^{32}$, Adorno souligne l'effet de choc ressenti lors du resurgissement d'une mode démodée: choc devant le «toujours identique toujours autre ", devant l'incompréhensible écoulement d'une histoire qui pourtant ne fait que se répéter. "L'humanité qui doute de sa possibilité de se reproduire, écrira finalement Adorno pour expliquer le phénomène, projette inconsciemment son désir de survivre dans la chimère des choses jamais connues, mais cette chimère ressemble à la mort ${ }^{33} \ldots$ »

30. Ibid., p. 316.

31. Ibid., p. 318-320. (Je souligne.)

32. "Le fait que quelque chose puisse encore changer dans la sphère d'une structure rationalisée suivant le modèle industriel est un paradoxe. Le principe même de la rationalité, dans la mesure où il applique des calculs commerciaux à des effets culturels, reste le toujoursmême. C'est pourquoi on est quelque peu choqué quand un produit du secteur de l'industrie culturelle se démode» (Theodor W. Adorno, "Les fameuses années vingt» dans Modèles critiques, Paris, Payot, 2003, p. 52.). Dans le même recueil, voir aussi le texte "Le progrès", p. 197: «Le processus vital lui-même se fige dans l'expression du toujours-semblable: d'où le choc que produisent les photographies du XIX ${ }^{e}$ siècle et maintenant déjà celles du début du $\mathrm{xx}^{\mathrm{e}}$ siècle. Le non-sens éclate du fait qu'il se passe quelque chose là où l'image dit qu'il ne peut plus rien se passer; son aspect terrifie." (Je souligne, dans les deux citations.)

33. Adorno, Minima Moralia, p. 320. (Je souligne.) 
La mort, écrivait Benjamin, constitue "le choc le plus profond de toute expérience individuelle ${ }^{34}$ » Freud, avant lui, disait éloquemment que "personne, au fond, ne croit à sa propre mort ${ }^{35}$ ». La réflexion sur la mort est un thème majeur dans la philosophie contemporaine, peut-être précisément parce que l'expérience concrète de la mort nous échappe comme jamais auparavant ${ }^{36}$. Benjamin liait à une certaine conscience de la mort la faculté de former des récits et de faire, à partir de la vie d'un homme, une histoire: la narration aurait reposé sur l'autorité dont se parait le mourant face aux vivants ${ }^{37}$. Poursuivant une réflexion similaire, Adorno écrit, dans Dialectique négative: "La mort devient aujourd'hui ce tout à fait étranger et ce, du fait de la décadence socialement déterminée de toute expérience continue. [...] Mort et histoire, surtout l'histoire collective de la catégorie d'individu, forment une constellation ${ }^{38}$.»

En même temps qu'éclate l'individu sous le coup des chocs incessants de la vie moderne, en même temps qu'il est réduit à l'impuissance par une organisation sociale dont la mesure le dépasse complètement s'étiole aussi sa capacité de faire face à la mort, à sa propre mort comme à celle des autres. Quand la vie, pour reprendre une expression déjà citée, est devenue une suite intemporelle de chocs, plus rien ne semble pouvoir la distinguer de cet ultime choc que sera la mort. Ou encore, cette dernière ne peut plus être perçue comme le point qui clôt l'histoire de la vie d'un homme lorsque celuici n'a plus que des réminiscences - ni souvenir ni oubli — des événements marquants de sa vie. En fait, ce sont tous les principaux thèmes qui ont jusqu'à présent animé cet essai sur la notion de choc qui semblent pouvoir être pensés en relation avec la mort: la mémoire et l'oubli, le rapport au temps et à l'espace, la perception des choses dans leur singularité, l'histoire. Pour l'illustrer, je me permets de citer longuement un fragment intitulé «De la théorie des revenants ", où Adorno voit dans notre relation aux morts un signe très révélateur de ce que nous sommes devenus:

La relation anormale avec les morts - le fait qu'ils sont oubliés et embaumés — est un des symptômes de la maladie qui, de nos jours, affecte l'expérience. On pourrait presque dire que c'est le concept même de vie humaine en tant qu'unité de l'histoire d'un individu qui est devenu caduc: la vie de l'individu

34. Benjamin, «Le narrateur », p. 160-161.

35. Freud, "Considérations actuelles sur la guerre et la mort", Essais de psychanalyse, p. 31.

36. "Jadis, il n'y avait guère de maison ni de pièce où quelqu'un ne fût mort. [...] Aujourd'hui les bourgeois vivent dans des lieux où personne n'est mort, froids habitants de l'éternité, qui, l'heure venue, iront mourir en maison de santé ou en clinique » (Benjamin, «Le narrateur", p. 152).

37. Ibid.

38. Adorno, Dialectique négative, Paris, Payot et Rivages, 2003, p. 448. 
n'est plus définie que par son contraire, la destruction, mais elle a perdu toute harmonie, toute continuité de la mémoire consciente et involontaire, elle a perdu toute signification. Les individus sont réduits à n'être plus que pure succession d'expériences instantanées qui ne laissent aucune trace, ou bien plus: leur trace est pour eux objet de haine parce qu'irrationnelle, superflue, littéralement dépassée. [...] En réalité, l'on inflige aux morts ce que les anciens Juifs considéraient comme la pire des malédictions: nul ne doit se souvenir de toi. Dans leur attitude envers les morts, les hommes laissent éclater leur désespoir de ne plus être capables de se souvenir d'eux-mêmes ${ }^{39}$.

Incapables de se souvenir d'eux-mêmes, les hommes refoulent la mort, liquident leur mémoire, abolissent l'histoire. Or de tels gestes d'évitement représentent, comme l'écrivait Adorno dans un passage déjà cité, autant de germes de destruction à venir. Le choc qui échappe tant à l'oubli salutaire qu'au souvenir salutaire, le choc qui marque le psychisme sans pouvoir être constitué en expérience devient le moteur d'une compulsion de répétition qui n'aura de cesse tant que l'événement traumatisant n'aura pas trouvé place dans la conscience. La mort, qui est l'inconcevable par excellence, sera d'autant plus facilement infligée aux autres que l'homme réussira à détourner continûment le regard de son horreur. On sait l'impact qu'a eu sur la réflexion d'Adorno, mais aussi sur la pensée occidentale dans son ensemble, le fait que des millions de Juifs ont pu être tués sous le régime nazi, et que des millions d'autres personnes ont péri dans les guerres du $\mathrm{xx}^{\mathrm{e}}$ siècle - et pourtant, des génocides sont encore perpétrés, et la guerre n'a toujours pas su être évitée. Adorno écrit :

La culpabilité de la vie qui, en tant que pur factum ravit déjà le souffle d'une autre vie, $[\ldots]$ ne peut plus être réconciliée avec la vie. Cette culpabilité se reproduit constamment parce qu'à aucun moment elle ne peut être totalement présente à la conscience. C'est cela et rien d'autre qui oblige à philosopher ${ }^{40}$.

Et Freud était vraisemblablement lui-même assez philosophe lorsque, à la fin d'une réflexion similaire, il écrivait, en modifiant le vieil adage: «Si vis vitam, para mortem. Si tu veux supporter la vie, organise-toi pour la mort $^{41}$.»

39. Adorno, Theodor W. et Max Horkheimer, La dialectique de la raison, Paris, Gallimard, 1974, p. 226-227. (Je souligne.)

40. Adorno, Dialectique négative, p. 441. (Je souligne.)

41. Freud, "Considérations actuelles sur la guerre et la mort», p. 46. Freud écrit aussi: «Ne devons-nous pas convenir qu'avec notre attitude de civilisé à l'égard de la mort nous avons, une fois encore, vécu psychologiquement au-dessus de nos moyens et ne devons-nous pas faire demi-tour et confesser la vérité ? Ne vaudrait-il pas mieux faire à la mort, dans la réalité de nos pensées, la place qui lui revient et laisser un peu plus se manifester notre attitude inconsciente à l'égard de la mort, que nous avons jusqu'ici si soigneusement réprimée. Cela ne semble pas être un progrès, plutôt sous maints rapports un recul, une régression, mais cela présente l'avantage de mieux tenir compte de la vraisemblance et de nous rendre la vie de nouveau supportable. Supporter la vie reste bien le premier devoir de tous les vivants.» 
C'est ni plus ni moins à un "nouvel impératif catégorique » que nous mène Adorno avec cette réflexion philosophique sur le choc et la nécessité d'enrayer cette folle compulsion de répétition. Sa maxime s'énonce comme suit: "Penser et agir en sorte que Auschwitz ne se répète pas, que rien de semblable n'arrive ${ }^{42}$." C'est à cet effet qu'Adorno parle, dans le passage précédent, d'une obligation de philosopher: d'une façon qu'on peut croire analogue à ce que fait la psychanalyse sur le plan individuel, la philosophie devient la thérapeutique qui doit permettre de prendre conscience, à l'échelle de la société, non seulement d'un traumatisme, mais aussi - et la tâche en est rendue d'autant plus ardue - d'une culpabilité généralisée. La philosophie doit faire reconnaître les chocs dans lesquels s'est décomposée l'expérience des individus modernes, et faire réfléchir sur leurs causes et leurs conséquences; elle doit faire voir lucidement à la fois la démesure des forces déployées par rapport aux capacités individuelles, et néanmoins l'impossibilité de s'abandonner à ces forces; elle doit remémorer l'horreur de la mort que l'on se targue d'avoir surmonté, mais qui ne cesse pourtant de nous hanter. La philosophie doit désactiver la compulsion de répétition afin de réactiver l'histoire.

Cette tâche échoie à la philosophie, et non à quelque autre discipline empirique, parce que le trouble qui est en jeu est un trouble éminemment philosophique, concernant tant la perception que le jugement. Les chocs reflètent la faillite de l'expérience, soit l'incapacité de saisir consciemment l'objet de l'expérience dans toute sa singularité et de le laisser agir en retour sur nous - cette incapacité étant, selon Benjamin, la marque "d'une perception dont le "sens de l'identique dans le monde" s'est aiguisé au point [...] [qu']elle parvient à standardiser l'unique ${ }^{43}$ ». Standardiser l'unique, c'est probablement le danger qu'associe Adorno à toute pensée conceptuelle, et le danger contre lequel la philosophie doit en propre nous défendre. La philosophie doit nous préserver de l'oubli de ce qui fait de chaque chose une chose unique, unique en elle-même et unique pour nous. L'expérience véritable, contrairement à une pure succession de chocs, est toujours liée à un contexte spatio-temporel, elle est historique dans sa structure même. Dès l'instant où la perception évacue ce contexte et ne saisit plus des objets que leur coquille vide - leur caractère standard, que ce soit une quantité d'excitation ou l'identité à un concept —, la faculté de produire des jugements moraux en est immédiatement affectée, croit Adorno. Il n'est dès lors pas étonnant de voir ce dernier évoquer la théorie du choc traumatique pour rendre compte du fait que, chez un même individu, une troublante insensibilité à des fautes morales graves puisse cohabiter avec d'amers remords

42. Adorno, Dialectique négative, p. 442.

43. Benjamin, L'œuvre d'art à l'époque de sa reproductibilité technique, p. 21. 
concernant des peccadilles ${ }^{44}$. Ces derniers, ces scrupules démesurés par rapport à leur objet, sont pour Adorno l'indice du fait que les décisions morales d'importance ont quitté le champ de l'expérience individuelle, de laquelle seule peut émerger un véritable sentiment moral. La disproportion avec laquelle des vétilles sont investies d'une culpabilité accablante témoigne de ce que ces dernières sont finalement devenues « un refuge pour l'expérience que repousse violemment l'ordre social objectivé ${ }^{45}$ » - elles concernent le seul domaine où les individus savent encore, par expérience, distinguer le bien du mal. Dans ces conditions, la seule morale qui puisse encore tenir, celle qui devra porter le lourd poids du nouvel impératif catégorique - ne pas répéter la catastrophe - sera celle qui saura se loger dans ce dernier refuge de l'expérience: une morale qu'Adorno qualifie de «micrologique ${ }^{46} »$.

L'une des premières tâches d'une morale micrologique, et probablement l'une des principales, serait de rendre possible quelque chose comme une expérience de la perte d'expérience, ou la conscience que ce monde qui devient abstrait et nous échappe ne va pas de soi. À propos de la façon dont il serait possible de s'acquitter de cette tâche, il semble que la notion de choc ait encore un ultime éclaircissement à nous donner. Dans l'essai où il commente la pièce Fin de partie de Samuel Beckett, Adorno écrit à un certain moment que «le langage de Beckett crée un choc salutaire au malade ${ }^{47}$ ». Le choc peut-il donc exercer un effet qui soit positif, salutaire? L'idée rappelle en tout cas la position de Benjamin sur le cinéma - qu'Adorno, d'ailleurs, avait vertement critiquée - selon laquelle le cinéma, en érigeant la perception traumatisante en principe formel, devait renforcer l'attention des masses et permettre à la perception humaine de relever les défis que lui posait le monde moderne ${ }^{48}$. Outre leur désaccord sur l'objet en question Adorno n'avait aucune foi dans les possibilités dites progressistes du cinéma,

44. "Comme le corps, l'organisme psychique est en mesure d'affronter des expériences d'un ordre de grandeur qui lui corresponde. Quand l'objet de l'expérience dépasse les mesures de l'individu, celui-ci ne la vit plus à proprement parler, mais l'enregistre immédiatement en s'en faisant une notion où n'intervient aucune intuition, comme quelque chose d'extérieur et d'incommensurable, à quoi il réagit avec une indifférence comparable à celle du choc catastrophique qui l'a atteint. Dans le domaine de la morale, il existe quelque chose d'analogue" (Adorno, Minima Moralia, p. 240).

45. Ibid., p. 241.

46. Ibid., p. 243.

47. Adorno, "Pour comprendre Fin de partie», Notes sur la littérature, Paris, Flammarion, 1993, p. 224. (Je souligne.)

48. Benjamin, L'œuvre d'art à l'époque de sa reproductibilité technique, p. 68 et suivantes. Sur le cinéma et l'entraînement qu'il prodigue, voir aussi «Sur quelques thèmes baudelairiens", p. 251. Adorno est loin de partager cette opinion sur le cinéma; dans Minima Moralia, sa réplique est cinglante: "Chaque fois que je vais au cinéma, j'en sors plus bête et pire que je n’y suis entré, malgré toute ma vigilance» (Minima Moralia, p. 26). 
ni de l'industrie culturelle en général -, il subsiste manifestement chez Adorno quelque chose de la dimension positive que prêtait Benjamin au choc: le choc, administré suivant la médecine d'un Beckett, conserve un potentiel critique.

Le choc qui est provoqué chez le spectateur par le langage de Beckett, pour poursuivre la citation précédente, s'énonce comme ceci: "en s'enten-

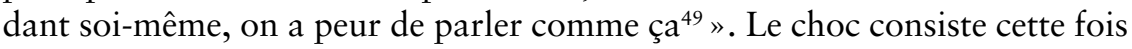
en un brusque soupçon à propos de la qualité de sa propre expérience, en un malaise soudain quant à l'inanité de ses propres paroles et conversations, en un doute poignant au sujet de la légitimité de l'ordre du monde. Plutôt que d'être l'occasion d'une diversion, le choc devient le moteur d'une réflexion. Dans cette optique, les aphorismes et fragments de l'œuvre philosophique d'Adorno apparaissent eux aussi comme autant de chocs qui doivent déstabiliser le lecteur et le renvoyer aux contenus de sa propre expérience ${ }^{50}$. La morale micrologique qui est appelée dans les Minima Moralia se présenterait ainsi comme une certaine théorie critique du choc: ni simulation d'une fausse continuité de l'expérience ni approbation de l'éclatement objectif de l'expérience en de multiples chocs.

\section{Bibliographie}

Adorno, Theodor W. Dialectique négative, trad. Gérard Coffin et al., Paris, Payot et et Rivages, 2003, 534 p.

—. La psychanalyse révisée, trad. Jacques Le Rider, Paris, Éditions de l'Olivier, 2007, $110 \mathrm{p}$.

- Minima Moralia. Réflexions sur la vie mutilée, trad. Éliane Kaufholz et JeanRené Ladmiral, Paris, Payot et Rivages, 2003, 357 p.

_. «Les fameuses années vingt» et «Le progrès» dans Modèles critiques, trad. Marc Jimenez et Éliane Kaufholz, Paris, Payot, 2003, pp. 51-59 et pp. 177-198.

—. "Pour comprendre Fin de partie» dans Notes sur la littérature, trad. Sibylle Muller, Paris, Flammarion, 1993, pp. 201-238.

—. et Max Horkheimer. La dialectique de la raison. Fragments philosophiques, trad. Éliane Kaufholz, Paris, Gallimard, 1974, 281 p.

Benjamin, Walter. «Le narrateur. Réflexions sur l'œuvre de Nicolas Leskov", "Sur quelques thèmes baudelairiens » et "Thèses sur la philosophie de l'histoire » dans CEuvres II - Poésie et Révolution, trad. Maurice de Gandillac, Paris, Denoël, 1971, pp. 139-169, pp. 225-275 et pp. 277-288.

—. L'œenvre d'art à l'époque de sa reproductibilité technique, trad. Maurice de Gandillac, Paris, Éditions Allia, 2006, 78 p.

Bernstein, Jay M. «Destruction of Aura, Destruction of Experience» dans Adorno. Disenchantment and Ethics, Cambridge, Cambridge University Press, 2001, pp. 111-120.

49. Adorno, «Pour comprendre Fin de partie», p. 224.

50. C'est ce que soutient Jay M. Bernstein, lorsqu'il écrit: «Only the "shock" of the fragment which breaks the uninterrupted flow of lived instant can synthetically produce the experience of the loss of experience» (Adorno. Disenchantment and Ethics, p. 120). 
Freud, Sigmund. «Considérations actuelles sur la guerre et la mort» et «Au-delà du principe de plaisir» dans Essais de psychanalyse, trad. André Bourguignon et al., Paris, Payot et Rivages, 2001, pp. 9-128.

Freud, Sigmund et Joseph Breuer. Études sur l'hystérie, trad. Anne Berman, Paris, PUF, 1956, $254 \mathrm{p}$.

Gadamer, Hans-Georg. Vérité et Méthode, Paris, Seuil, 1996, 534 p.

Jay, Martin. "Is Experience Still in Crisis? Reflections on a Frankfurt School Lament» dans The Cambridge Companion to Adorno, Tom Huhn (dir.), Cambridge, Cambridge University Press, 2004, pp. 129-147.

Marinetti, Filippo Tommaso. Tuons le clair de lune! Manifestes futuristes et autres proclamations, Paris, Mille et une nuits, 2005, 79 p.

Marx, Karl. Le Capital, livre I, trad. Étienne Balibar et al., Paris, PUF, coll. Quadrige, 1993, 940 p.

Sherratt, Yvonne. "The Dialectic of Enlightment: a Contemporary Reading" dans History of the Human Sciences, vol. 12, n 3, 1999, pp. 35-54.

Whitebook, Joel. «Weighty Objects. On Adorno's Kant-Freud Interpretation» dans The Cambridge Companion to Adorno, Tom Huhn (dir.), Cambridge, Cambridge University Press, 2004, pp. 51-78.

Wolin, Richard. "Benjamin's Materialist Theory of Experience " dans Theory and Society, $\mathrm{n}^{\circ} 11,1982$, pp. 17-42. 